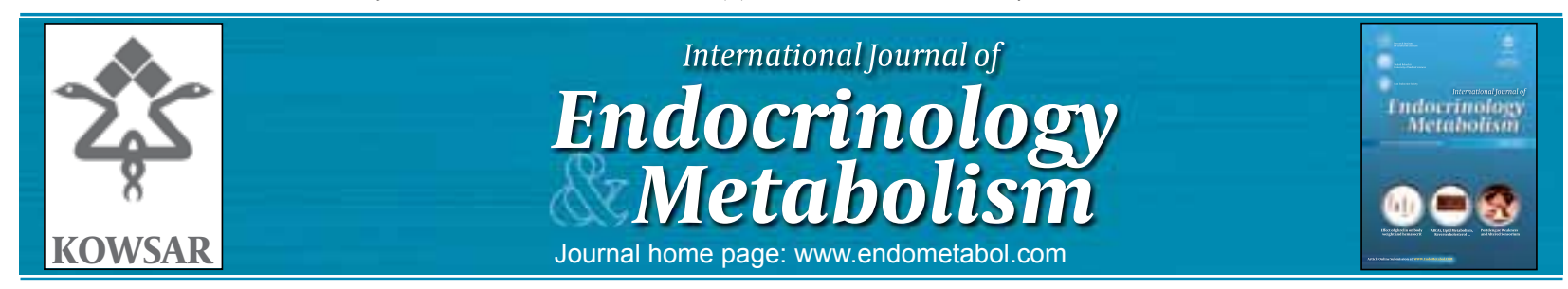

\title{
Testosterone Replacement Helps
}

\author{
Anna Maria Aloisi $1^{1^{*}}$ \\ ${ }^{1}$ Department of Physiology, University of Siena, Siena, Italy
}

\section{A R T I C L E I N F O}

\section{Article Type:}

Letter to Editor

Article history:

Received: 03 Sep 2010

Revised: 22 Sep 2010

Accepted: 06 Dec 2010

\section{Keywords:}

Testosterone

Hormon replacement therapy

Hypogonadism

\section{Dear Editor,}

A recent paper in the International Journal of Endocrinology And Metabolism (1) dealt with hormone replacement therapy carried out in hypogonadotropic hypogonadal $(\mathrm{HH})$ men. The authors described a successful procedure involving the use of gonadotropin-like substances and testosterone ( $\mathrm{T}$ ) to fight infertility in $\mathrm{HH}$ men. The findings of the study suggested that the procedure could lead to successful sexual relationships and conception. Despite the important role played by these hormones, the design of experiments aimed at better defining guidelines for $\mathrm{T}$ use in various pathological conditions, other than those related to reproductive functions, is still rare. In the present letter, I wish to underline some aspects of this attitude.

Hormones are defined as substances secreted by glands into the blood (or released into the interstitial space) and capable of acting on specific receptors located in target organs. In addition to the various glands known to possess the biosynthetic enzymes required to produce the hormones, other tissues act as mediators of hormonal functions; the cells in these tissues take up the prohormones from the blood and transform them into the final

\footnotetext{
* Corresponding author at: Anna Maria Aloisi, Department of Physiology, Section of Neuroscience and Applied Physiology, University of Siena, Siena, Italy. Tel:+39-0577234103, Fax:+39-0577234037, E-mail: aloisi@unisi.it DOI: 10.5812/Kowsar.1726913X.1929

Copyright $\odot$ 2011, BRCGL, Published by Kowsar M.P.Co. All rights reserved.
}

- Please cite this paper as:

Aloisi AM. Testosterone Replacement Helps. Int J Endocriol Metab. 2011;9(1): 280-1. DOI: 10.5812/Kowsar.1726913X.1929

๑ 2011 Kowsar M.P.Co. All rights reserved.

useful product.

Among the endocrine systems of the body, those related to the hypothalamus-pituitary (HP) axis have been particularly well studied because of their direct involvement in reproductive functions. The HP-gonadal axis in men, and particularly in women, has been extensively studied in relation to the physiology of fertility. The possibility of altering these hormonal functions has led to the development of a number of therapeutic approaches based on hormonal manipulation in women; these include the need to prevent pregnancy (with pills), the need to induce it (in vitro fertilization), and the need to maintain the reproductive status as long as possible to avoid menopause. Synthetic estrogens and progestins were developed to help in these conditions. Thus, hormone therapy in women is not new. However, the use of hormonal treatments in men has never been considered "routine." This is evident from the effort to counteract pregnancy only via manipulation of female physiology. Attempts to develop a male birth control pill have only been made in recent years (2).

Androgens and estrogens are naturally present in men and are known to play a role in many body functions, in addition to reproduction. Moreover, despite the official ban on the use of hormones as doping, many men commonly use hormone-like products to improve body strength and performance a procedure called "doping." These practices mostly involve the use of androgens, in particular T. Hence, among men, the general attitude is to "not con- 
sider" hormones for medical treatment, whereas unofficially a high percentage of men wish to alter their physical power by using androgens. Another aspect of this issue is that doping is not socially accepted and can be punished under law. Therefore, the idea of taking $\mathrm{T}$ has always had a somewhat negative connotation, and this has resulted in the use of $\mathrm{T}$ in very few cases in which hypofunction of the testes was a clear clinical sign. The low levels of these hormones in the blood also make it difficult to regulate their levels. The level of $\mathrm{T}$ is difficult to determine. Like other steroids, it binds to proteins and exhibits properties characteristic of steroids; therefore, complicated laboratory procedures have to be performed to determine its levels. However, routine examinations can give only approximate values when very low levels have to be determined (3). Androgens, particularly T, play a significant role in bone and muscle remodeling, erythropoietin induction, libido enhancement, and the development and maintenance of secondary sexual characteristics. However, the levels of $\mathrm{T}$ are not routinely determined in men or women. Outside the obvious pathologies related to reproductive functions, it is uncommon to see general physicians order this exam among those necessary to obtain a basic picture of the patient's status, unlike other hormones such as thyroid hormones. Another aspect of $\mathrm{T}$ use that has probably had a negative influence on its popularity is the difficulty of its administration. Studies in the literature have listed many reasons why oral administration or, more recently, patch use of T should be avoided, not least of which is the uncertainty about the negative effects of its metabolism by the liver. At present, conventional wisdom still considers $\mathrm{T}$ administration risky despite the availability of new safe tools for administering this hormone, e.g., long-term intramuscular administration. Indeed, much effort has been made to obtain a T products able to regularly supply the $\mathrm{T}$ dose without the many known side effects (4). On the other hand, there is an interesting aspect of the clinical approach to this problem that has rarely been considered the different conditions of the subjects when taking T. In general, when a drug is tested, its interactions with other drugs must be determined and/or the clinical conditions of the liver and kidneys must be tested. Recently, consideration has been given to the possible interactions of $\mathrm{T}$ with other drugs, particularly opioids, as described by Azizi F. (5). Opioids, particularly morphine, have been shown to significantly slow the increase in blood levels of $\mathrm{T}$ in patients having chronic non-malignant pain and implanted with an intrathecal. catheter or chronically treated with opioids. The patients included in the study (6) received a daily application of testosterone gel but showed the first sign of efficacy a few days after the beginning of treatment. Appetite and other physical responses immediately increased. However, in patients with basal T levels of $0.5-0.9 \mathrm{ng} / \mathrm{ml}$, the T concentration in the blood did not follow the expected time course, and the highest value was noted after ca 1 year of treatment (6). Further experiments conducted to explain these results showed that opioids, as well as other drugs, affected the T system at different levels, either increasing its catabolism into estradiol or DHT(dihydrotestosterone) or decreasing its production through direct (toward the gonads) or indirect (toward the hypothalamus) inhibitory action (7).

In conclusion, much effort must still be made to follow the few examples in the literature in which hormone replacement therapy has been shown to greatly improve the quality of life of patients.

\section{Financial Disclosure}

None declared.

\section{References}

1. Farshchi H, Shahnazi A, Azizi F. Effects of Testosterone and Gonadotropin Therapy in Men with Hypogonadotropic Hypogonadism. Int $J$ Endocriol Metab. 2009;7(4):242-7.

2. Aitken RJ, Hughes LM, Griffith R, Baker MA. Bridging the gap between male and female fertility control; contraception-on-demand. Contraception. 2008;78(4):S28-S35.

3. Savolainen K, Kiimamaa R, Halonen T. High-throughput analysis of testosterone in serum samples by on-line solid phase extraction liquid chromatography-tandem mass spectrometry. Clin Chem Lab Med. 2011[Epub ahead of print].

4. Handelsman DJ. Androgen misuse and abuse. Best Pract Res Clin Endocrinol Metab. 2011;25(2):377-89.

5. Azizi F, Vagenakis AG, Longcope C, Ingbar SH, Braverman LE. Decreased serum testosterone concentration in male heroin and methadone addicts. Steroids. 1973;22(4):467-72.

6. Aloisi AM, Ceccarelli I, Carlucci M, Suman A, Sindaco G, Mameli S, et al. Hormone replacement therapy in morphine-induced hypogonadic male chronic pain patients. Reprod Biol Endocrinol. 2011;9:26.

7. Aloisi AM, Ceccarelli I, Fiorenzani P, Maddalena M, Rossi A, Tomei $\mathrm{V}$, et al. Aromatase and 5-alpha reductase gene expression: modulation by pain and morphine treatment in male rats. Mol Pain. 2010;6:69. 